\title{
Studies on the Control of Postharvest Diseases of Muskmelons and the Induction of Resistance Related Enzymes and Defense Substances by Salicylic Acid Treatment
}

\author{
Jing Yuanyuan, Li Na, Li Lihua, Zhu Xuan* \\ Department of Food Science, Xinjiang Agricultural University, Urumqi, China \\ Email address: \\ 785738415@qq.com (Jing Yuanyuan),zx9927@126.com (Zhu Xuan) \\ ${ }^{*}$ Corresponding author
}

\section{To cite this article:}

Jing Yuanyuan, Li Na, Li Lihua, Zhu Xuan. Studies on the Control of Postharvest Diseases of Muskmelons and the Induction of Resistance Related Enzymes and Defense Substances by Salicylic Acid Treatment. Science Discovery. Vol. 4, No. 4, 2016, pp. $226-231$.

doi: 10.11648/j.sd.20160404.14

Received: June 30, 2016; Accepted: July 1, 2016; Published: July 20, 2016

\begin{abstract}
The enzymes activity related muskmelon disease resistance by salicylic acid treatment in pro-harvest muskmelon fruits was investigated in this paper. The plants of "Jin tian mi" muskmelon as test materials, muskmelons were sprayed at four growing seasons: young fruit period (two weeks after flowering), ruit enlarging period (three weeks after flowering), netting period (four weeks after flowering) and $48 \mathrm{~h}$ before picking by the $1.0 \mathrm{mmol} / \mathrm{L}$ salicylic acid solution, respectively. The harvest fruits were storage at $7^{\circ} \mathrm{C}$ after precooling. The incidence of disease, disease index; the content of total phenols, flavonoids and lignin of muskmelon, CHT, GLU and PAL activities of muskmelon were measured during the storage every 7 days. The results showed that: compared with the control, the disease time of muskmelon was delaywd, fruit disease incidence and disease index were decreased, chitinase and $\beta$-1,3-glucanase were induced, phenylalanine ammonia lyase activity was increased, and the total phenolic, flavonoid, lignin content were better maintain by $1.0 \mathrm{mmol} / \mathrm{L}$ salicylic acid treatment. It is suggested that the increase of the related enzymes activity induced by salicylic acid were closely related to the increase of disease resistance in pro-harvest muskmelons.
\end{abstract}

Keywords: Salicylic Acid, Muskmelon, Resistance Related Enzymes, Induction, Disease Resistance

\section{采前水杨酸处理对甜瓜采后病害的控制及抗性相关酶和 防卫物质诱导的研究}

敬媛媛, 李娜, 李丽花, 朱璇*

食品科学与药学学院, 食品科学系, 新疆农业大学, 乌鲁木齐, 中国

邮箱

785738415@qq. com(敬媛媛), zx9927@126.com(朱璇)

摘要：为了探究采前水杨酸处理诱导甜瓜果实采后抗病性及相关酶活性的变化，以 “金甜蜜” 甜瓜果实为试材，采用 1.0 $\mathrm{mmol} / \mathrm{L}$ 的水杨酸溶液分别在甜瓜幼果期(花后2周)、膨大期(花后3周)、网纹形成期(花后4周) 及采收前48 h的四个生长期进 行喷施处理, 采摘后置于 $7^{\circ} \mathrm{C}$ 咜藏。定期对甜瓜果实的发病率、病情指数、总酚含量、类黄酮含量、木质素含量、苯丙氨酸 解氨酶活性、几丁质酶活性、 $\beta-1,3$-葡聚糖酶活性进行测定。试验结果表明: $1.0 \mathrm{mmol} / \mathrm{L}$ 水杨酸处理能够推迟甜瓜果实的 发病时间, 降低果实发病率和病情指数; 诱导甜瓜果实几丁质酶、 $\beta-1,3$-葡聚糖酶、苯丙氨酸解氨酶活性的升高; 较好地 
维持果实总酚、类黄酮、木质素的含量。说明采前水杨酸处理诱导果实抗性相关酶活性的升高与提高果实采后抗病性密切 相关。

关键词: 水杨酸, 甜瓜, 抗性相关酶, 诱导, 抗病性

\section{1. 引言}

新疆（中国）甜瓜以其 “品质优良、风味独特”而驰 名中外。新疆是中国甜瓜的主产区之一，甜瓜在中国栽培 历史悠久，栽培面积和产量均居世界前列。但甜瓜果实糖 度较高, 水分含量较大, 采后代谢旺盛, 加之采收期相对 集中且正值高温夏季, 极易受外界病菌侵染而发生腐烂变 质, 造成巨大的经济损失 [1]。引起甜瓜采后腐烂的病原菌 主要有链格狍 (Alternaria alternata)、镰刀菌 (Fusarium $s p$.)、粉霉病 (Trochothecium roseum) 、软腐病 (Rhizopus sp. ) 、青霉病（Penicillium sp. ） [2], 其中, 链格孢 (Alternaria alternata) 和镰刀菌（Fusarium sp.）是引 起甜瓜低温拒运过程中腐烂的优势病原菌, 并且在甜瓜生 长期间就可能会侵染果实, 以潜伏状态存在于果皮组织中, 加剧果实采后腐烂 [3]。化学杀菌剂虽然可有效控制甜瓜的 采后病害 [4], 但由于残留问题、环境污染及诱导病原菌产 生抗药性等问题而受到限制 [5]。因此, 寻求新型安全有效 的防腐保鲜措施已成为甜瓜咜运产业中函待解决的问题。

水扬酸 (salicylic acid, SA) 是一种小分子酚类物质, 普遍存在于植物体内。SA也是植物受病原菌浸染后活化一 系列防卫反应信号传导途径中的重要组成部分 [6-7], 还能 够作为信号分子诱发系统获得性抗性 (Systemic Acquired Resistance, SAR), 而SAR能够诱导植物持续抵御病原微生 物的侵害 [8]。研究表明, 外源SA不仅能够诱导多种果蔬对 真菌、细菌和病毒等病原菌产生局部或系统抗性 [9], 还能 够提高果蔬的采后抗病性。范存斐 [10]等研究发现 $\mathrm{SA}$ 处理 通过对厚皮甜瓜苯丙烷代谢的调控抑制其采后病害, 外源 $\mathrm{SA}$ 处理还能诱导芒果 [11]、苹果 [12]、马铃薯 [13]等果蔬 抗病能力的提高, 控制果蔬的采后腐烂。

目前, 关于SA采前处理的应用较少, 采前SA处理诱导 甜瓜采后抗病性的研究鲜见报道。因此, 本试验采用 1.0 $\mathrm{mmol} / \mathrm{L}$ SA在甜瓜采前进行喷施处理, 以探讨 SA处理对甜瓜 采后抗病性及相关酶活性的影响, 为采前SA处理对甜瓜采 后病害的控制及其作用机理的研究提供理论依据。

\section{2. 材料与方法}

\section{1. 试验材料}

甜瓜品种为 “金甜蜜” 。

\section{2. 试验方法}

试验于五家渠市 103 团7连进行, 参照李梅 [14]等的方 法并修改, 选择地势、光照、通风一致的地块, 每处理两 垄进行区组试验设计。分别在甜瓜幼果期 (花后2周)、果 实迅速膨大期(花后 3 周)、网纹形成期(花4周)和采前 $48 \mathrm{~h}$
四个时期用手动式喷雾器均匀喷酒浓度为 $1.0 \mathrm{mmol} / \mathrm{L}$ 的 $\mathrm{SA}$, 并以清水处理为对照。每升药液处理甜瓜 35 株, 每处理 70 株, 重复3次。采收后的果实选择无病虫害和机械损伤、 果柄处带 $\mathrm{T}$ 形蔓的果实, 外套发泡塑料网套, 防止运输过 程中甜瓜的碰撞损伤, 随后将果实装箱运置新疆农业大学 食品科学与药学学院冷库中, 低温 $\left(7 \pm 1^{\circ} \mathrm{C}, \mathrm{RH} 85 \%\right)$ 败藏, 贮藏期间每 $7 d$ 对各项指标进行测定。

\section{3. 测定项目及方法}

\subsection{1. 自然发病率测定}

采后病害调查: 果实采收后, 发泡网袋单果包装, 装 入瓦楞纸板箱, 每箱 4 个瓜, 汽车当天运抵实验室, 置于 冷库 $\left(7 \pm 1^{\circ} \mathrm{C}, 85 \% \mathrm{RH}\right)$ 败藏, 观察采后病害的发病情况和 病情指数。每处理 6 个瓜, 重复 3 次。采后病害分级参照 Huang [15] 等的方法。方法: 0级-无任何症状, 1级-一个 病斑, 直径小于 $1 \mathrm{~cm}, 2$ 级 - 直径在 $1 \sim 3 \mathrm{~cm}$ 的病斑一个或直 径小于 $2 \mathrm{~cm}$ 的病斑 2 个, 3 级-直径在 $3 \sim 5 \mathrm{~cm}$ 的病斑一个或直 径小于 $2 \sim 3 \mathrm{~cm}$ 的病斑两个, 4 级-病斑直径大于 $5 \mathrm{~cm}$ 或 3 个以 上的病斑。发病瓜定义为出现二级及以上级别的病害。

发病率（\%) =发病瓜个数/总瓜数 $\times 100 \%$

\subsection{2. 病情指数的计算方法及数据处理}

按下列公式计算病情指数:

$$
\text { 病情指数 }=\sum \frac{(\text { 发病瓜数 } \times \text { 发病级别 })}{(\text { 总瓜数 } \times \text { 最高级别 })}
$$

\subsection{3. 总酚、类黄酮含量测定}

参照曹建康 [16]等的方法。

\subsection{4. 木质素含量的测定}

参照朱海英的方法并修改 [17], 取甜瓜果肉 $12 \mathrm{~g}$, 于 研针中研碎后, 用 $12.0 \mathrm{~mL}$ 的 $70 \%$ 硫酸溶解均浆液于 $100 \mathrm{~mL}$ 的小烧杯中。将小烧杯在 $40^{\circ} \mathrm{C}$ 下恒温水浴 $1 \mathrm{~h}$, 其间不断摚 拌。反应一小时后, 用 $200 \mathrm{~mL}$ 蒸馏水冲洗均浆液于 $500 \mathrm{~mL}$ 三角瓶中, 用牛皮纸（或报纸）、纱布封口后, 于立式压 力蒸汽灭菌器中 $121^{\circ} \mathrm{C}$ 保温 $1 \mathrm{~h}$, 然后将所得热溶液通过 砂心漏斗进行真空抽滤, 并用热水冲洗残渣。最后将所得 残渣于 $60^{\circ} \mathrm{C}$ 的电热恒温鼓风干燥箱中烘至恒重后用电子 天平称量所得残渣重。木质素含量 $(\%)=$ 残渣重/样品质量 $\times 100 \%$, 测定 3 次重复。如上, 重复三次平行实验, 取平均 数作为木质素含量值。

\subsection{5. 苯丙氨酸解氨酶活性测定}

参照曹建康 $[16]$ 等的方法。 


\subsection{6. 几丁质酶的活性的测定}

参照曹建康 [16]等的方法。

\subsection{7. $\beta-1,3$-葡聚糖酶活性测定}

参照曹建康 $[16]$ 等的方法。

\section{4. 数据统计}

全部试验数据用Microsoft Excel 2007和DPS 7.55数 据处理系统进行统计处理, 计算标准偏差 $( \pm \mathrm{SE})$ 或进行 Duncan’s多重差异显著分析。

\section{3. 结果与分析}

\section{1. SA处理对甜瓜发病率的影响}

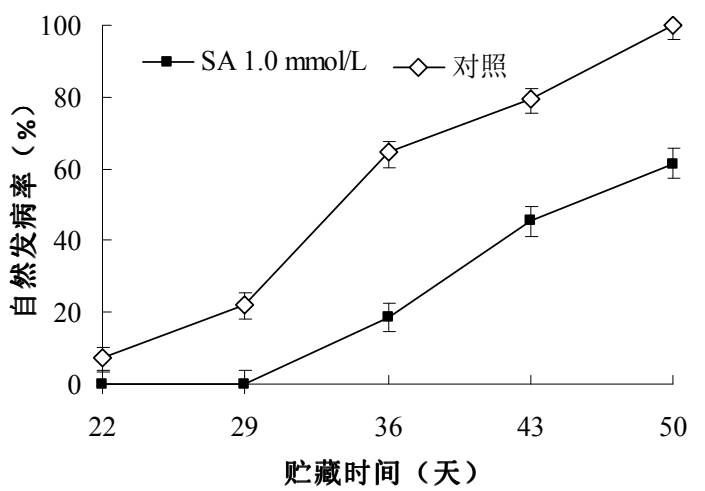

图1 SA处理对甜瓜发病率的影响。

在䛎藏18天时，对照果实开始发病，而 $1.0 \mathrm{mmol} / \mathrm{L} \mathrm{SA}$ 处理在聍藏31天时才开始发病。如图1所示, 咜藏50天时, $1.0 \mathrm{mmol} / \mathrm{LSA}$ 处理和对照果实的发病率分别是 $60 \% 、 100 \%$, $1.0 \mathrm{mmol} / \mathrm{L} \mathrm{SA}$ 处理发病率比对照低 $40 \%(p<0.05) 。 1.0$ $\mathrm{mmol} / \mathrm{L}$ 不仅可以降低甜瓜果实的发病率，且可以推迟甜瓜 果实的发病时间。说明采用 $1.0 \mathrm{mmol} / \mathrm{L} \mathrm{SA}$ 能明显抑制甜 瓜果实聍藏期间病害的发生。

\subsection{SA处理对甜瓜病情指数的影响}

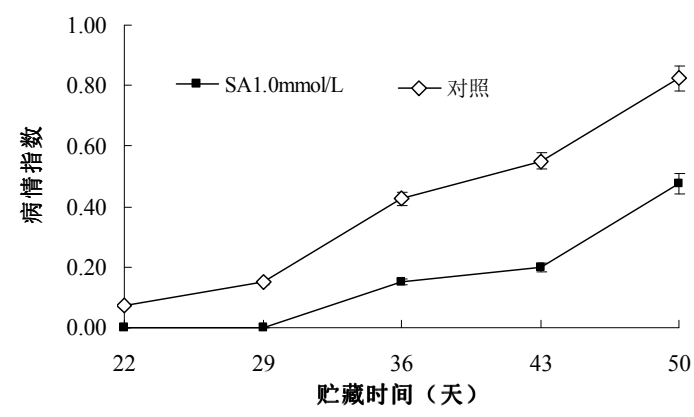

图2 SA处理对甜瓜迄藏期间病情指数的影响。

如图2所示, 随着咜藏时间的延长，果实的病情指数 不断上升, 且在整个陉藏过程, 对照果实的病情指数明显 高于SA处理组。在它藏50天时, 对照果实和SA处理果实的 病情指数分别为 $82.5 \%$ 和 $47.5 \%$, SA处理的病情指数比对照
低了 $35 \% （ p<0.05 ） 。$ 说明 $\mathrm{SA}$ 处理可以降低甜瓜果实的病 情指数。

\section{3. SA处理对甜瓜总酚和类黄酮的影响}

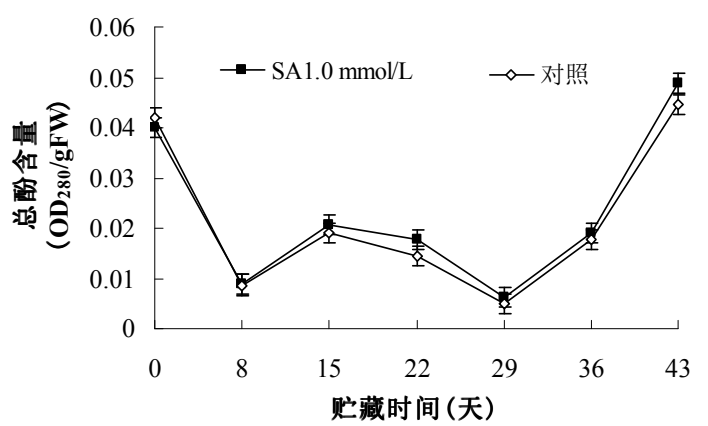

图3 SA处理对甜瓜总酚含量的影响。

如图3所示, 甜瓜果实采收时, 对照果实总酚含量比 $\mathrm{SA}$ 处理略高。但是在整个败藏过程中, 经SA处理果实的总 酚含量高于对照果实。这说明, 在它藏前期, 采前 $\mathrm{SA}$ 处理 有助于败藏过程中总酚含量的保持; 而在尖藏后期, $\mathrm{SA}$ 处 理有利于总酚含量的积累。

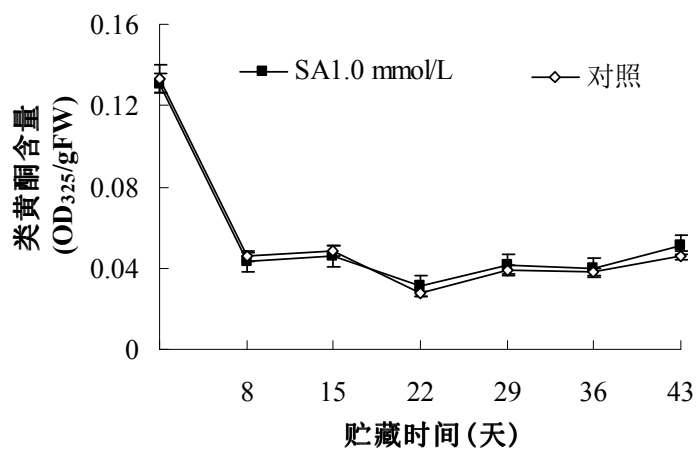

图4 SA处理对甜瓜类黄酮含量的影响。

如图4所示, 在栬藏前 8 天内, 类黄酮含量显著下降, 而在咜藏 8 天以后, 类黄酮整体趋势呈先下降后升高的趋 势, 但变化幅度不显著。贮藏 22 天以前对照果实的类黄酮 含量比SA处理的果实高, 而 22 天以后, 经 $\mathrm{SA}$ 处理的类黄酮 含量比对照果实高。由此可见, SA处理可以诱导类黄酮的 含量的积累。

\section{4. SA处理对甜瓜木质素的影响}

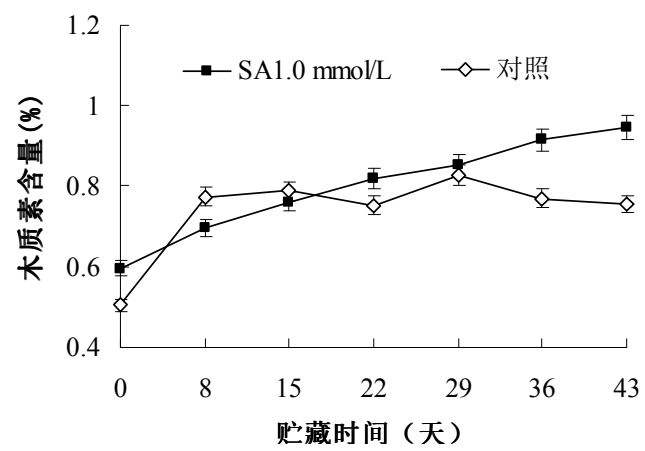

图5 SA处理对甜瓜木质素含量的影响。 
如图5所示, 在整个贮藏过程中, 随着贮藏时间的延 长, $\mathrm{SA}$ 处理果实的木质素含量逐渐增加; 而对照果实在咜 藏前 8 天上升较快, 之后趋于平稳状态。在栬藏 22 天后, 经SA处理果实的木质素含量高于对照组。败藏43天时, 1.0 mmol/L SA处理和对照果实的木质素含量分别是 $0.947 \%$ 、 $0.755 \%(p<0.05)$ 。说明 $1.0 \mathrm{mmol} / \mathrm{L} \mathrm{SA}$ 处理能够诱导甜瓜 果实中木质素含量的积累。

\section{5. SA处理对甜瓜苯丙氨酸解氨酶的影响}

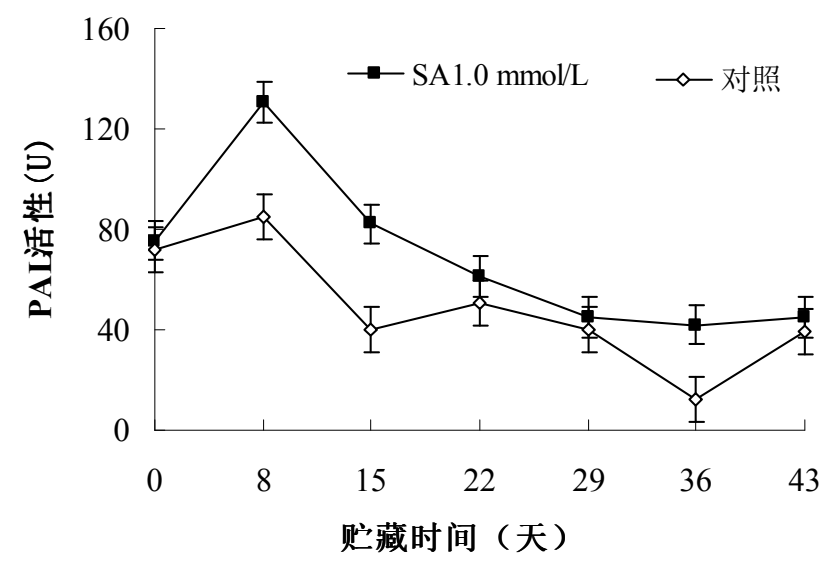

图6 SA处理对甜瓜苯丙氨酸解氨酶活性的影响。

如图6所示, 赔藏过程中PAL活性整体呈先上升后下降 趋势。在整个䛎藏过程中, 经 $1.0 \mathrm{mmol} / \mathrm{L} \mathrm{SA}$ 处理果实的 PAL活性明显高于对照果实, 在咜藏第8天和第 15 天时, SA 处理果实的 PAL 活性分别是对照果实的 1.5 倍和 2 倍 $(p<0.05)$ 。说明采前 $1.0 \mathrm{mmol} / \mathrm{L} \mathrm{SA}$ 处理能够诱导甜瓜 果实采后PAL活性的升高。

\subsection{SA处理对甜瓜几丁质酶活性的影响}

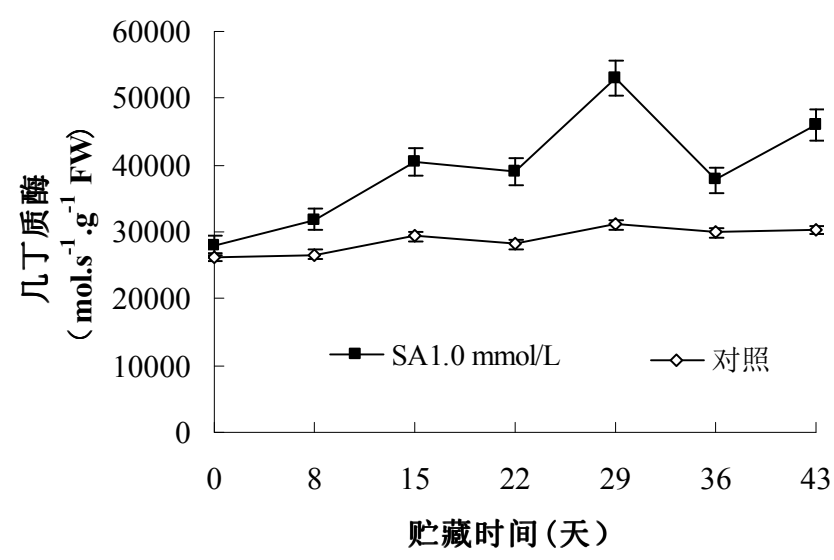

图7 SA处理对甜瓜几丁质酶活性的影响。

如图7所示, 随着败藏时间的延长, 甜瓜果实的CHT活 性活性总体上呈上升趋势, 对照组果实变化幅度较小。SA 处理组CHT活性在整个败藏过程期间明显高于对照果实。 在贮藏第29天时, SA处理组CHT活性比对照组高了 $70.7 \%$ $(p<0.01)$ 。说明SA处理能够诱导甜瓜果实采后CHT活性 的上升。

\section{7. $S A$ 处理对甜瓜 $\beta-1,3$-葡聚糖酶活性的影响}

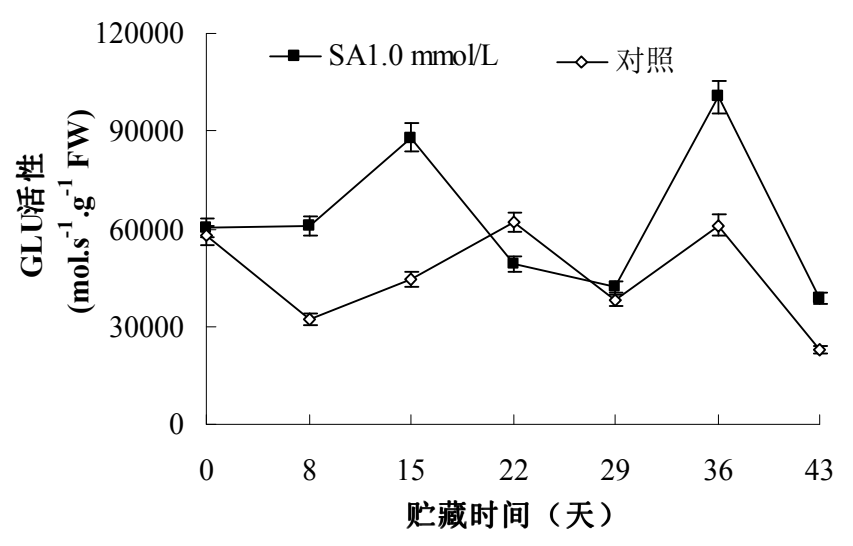

图8 SA处理对甜瓜 $\beta-1,3$-葡聚糖酶活性的影响。

$\beta-1,3$ 葡聚糖酶（GLU）在高等植物中普遍存在，能 够降解真菌细胞壁中的 $\beta-1,3$-葡聚糖, 该酶还能使真菌 细胞释放出 $\beta-1,3$ 葡聚糖来源的抗性诱导因子, 从而激活 防卫相关酶系统。如图8所示, 贮藏过程中, SA处理组甜 瓜果实的GLU活性整体上高于对照果实。在捾藏第 36 天时, SA处理组GLU活性比对照组高了 $64.2 \%(p<0.05)$, 说明SA 处理能够诱导甜瓜果实采后GLU活性的增加。

\section{4. 讨论与结论}

本研究表明, $1.0 \mathrm{mmol} / \mathrm{L} \mathrm{SA}$ 处理可以推迟甜瓜果实 的发病时间, 降低其发病率和病情指数。这与SA处理对鸭 梨 [18]、厚皮甜瓜 $[10]$ 和甜樱桃 [19] 上的结果相一致。 Poole [20]和Davarynejad [21] 等研究发现, 采前或采后SA 处理猕猴桃果实, 都能够诱导果实产生抗病性。 Babalar [22]等的研究也表明, 在 1-2 mmol/L的浓度范围 内, SA处理可有效降低真菌引起的草莓类果实的腐烂。本 试验是在甜瓜采前的 4 个重要时期用 $1.0 \mathrm{mmol} / \mathrm{L} \mathrm{SA}$ 进行喷 施处理, 不断地诱导果实抗病能力的增强, 从而使果实采 后具有较强的抗病性, 而且这种抗病性具有一定的持久性。

CHT和GLU是两类重要的病程相关蛋白, 被认为是植物 系统获得抗病性 (SAR) 的标志 [23]。CHT和GLU水解几丁 质和 $\beta-1,3$-葡聚糖的过程中, 由真菌细胞壁释放出来的 寊糖能够作为植物多种抗病反应的激发因子, 诱导植物的 全面防卫反应 [24-25]。本研究表明, 采前 $1.0 \mathrm{mmol} / \mathrm{L} \mathrm{SA}$ 处理可以诱导甜瓜果实在采后整个陉藏过程中CHT和GLU 活性均显著高于对照组, 这可能是SA处理诱导甜瓜启动自 身抗病系统而产生抗病性的机制之一。这与张衍荣 [26]对 豇豆的研究中经SA处理豇豆感病品种后可以诱导GLU和 CHT活性升高的结果相一致。

酚类物质本身具有抗菌素的性质, 并且由酚类物质氧 化而成的醌类物质是潜在的抗病因子。PAL与酚类化合物 的合成有着十分密切的联系, 同时也是木质素合成的关键 酶和限速酶, 与SAR的表达存在相关性 [27-28]。木质素不 仅对病原微生物的侵害起屏障作用, 还能与低分子量酚类 前体物质多聚作用产生游离基针化病原真菌细胞。王媛 [29] 等的研究表明, SA处理可以降低拟南芥灰霉病 
(Botrytiscinerea) 的发生率，提高拟南芥叶片PAL的活性， 并诱导木质素大量积累。张帆 [30]等研究也表明, 采用 1.0 $\mathrm{mmol} / \mathrm{L}$ SA处理树莓, 在拒藏后期可以提高总酚、类黄酮 等酚类物质的含量。在甜樱桃 [19]、芒果 [31]中的研究表 明, SA处理可显著增加果实PAL的活性从而降低其采后褐 腐病和炭瘨病的发生。本研究中, 采前 $1.0 \mathrm{mmol} / \mathrm{L} \mathrm{SA}$ 处 理可诱导PAL活性在整个咜藏过程中都高于对照果实; 总 酚含量在咜藏15天后高于对照果实; 类黄酮和木质素的含 量都有不同程度的提高, 这也是SA处理能够增强果实采后 抗病性的主要原因之一。

综上所述, $1.0 \mathrm{mmol} / \mathrm{L}$ 水杨酸处理能够推迟甜瓜果实 的发病时间, 降低果实发病率和病情指数; 诱导甜瓜果实 几丁质酶、 $\beta-1,3$ - 葡聚糖酶、苯丙氨酸解氨酶活性的升 高; 较好地维持果实的总酚、类黄酮、木质素的含量。说 明采前水杨酸处理诱导果实抗性相关酶活性的升高与提 高果实采后抗病性密切相关。

\section{参考文献}

[1] 王建玉, 邓志斌, 王志鹏. 新疆五家渠地区甜瓜滴灌简易栽 培技术 $[J]$. 中国瓜菜, 2015(1):68-68.

[2] Bi Y, Li Y, Ge Y, et al. Induced Resistance in Melons by Elicitors for the Control of Postharvest Diseases [M]// Postharvest Pathology. Springer Netherlands, 2009: 31-41.

［3］葛永红, 毕阳, 马凌云. 黄河蜜甜瓜致病真菌潜伏侵染的时 期与途径 $[J]$. 中国西瓜甜瓜，2005，(3) : 1-3.

４４］马凌云, 赵亮. 嘧菌酯处理对 “银帝” 甜瓜采后病害控制的 研究 [J]. 信阳农业高等专科学校学 报, 2009, 19 (4) : 118-119.

[5] Terry L A, Joyce D C. Elicitors of induced disease resistance in postharvest horticultural crops: a brief review. Postharvest Biology and Technology, 2004, 32: 1-13.

[6] Sendon P M, Seo H S, Song J T. Salicylic Acid Signaling: Biosynthesis, Metabolism, and Crosstalk with Jasmonic Acid[J]. Journal of the Korean Society for Applied Biological Chemistry, 2011, 54(4) : 501-506.

[7] Kumar D. Salicylic acid signaling in disease resistance $[J]$. Plant Science, 2014, 228: 127-134.

[8] 张艳秋, 崔崇士. 植物系统获得性抗性研究进展 [J]. 东北农 业大学学报, 2008, 39 (12): 113-117.

[9] 龙亚芹, 王万东, 王美存, 等. 水杨酸 (SA) 诱导植物对病虫害 产生抗性及作用机制研究 [J]. 热带农业科 学, 2009, 29 (12) :46-50.

[10］范存斐, 毕阳, 王云飞, 等. 水杨酸对厚皮甜瓜采后病害及苯 丙烷代谢的影响 [J]. 中国农业科学2012, 45(3) :584-589
[11] 杨冬平, 高兆银, 李敏, 等. 水杨酸结合超声波处理对芒果采 后抗病性的影响 [J]. 热带作物学报, 2014, 35 (5) : 974-979.

[12] 王芳芳. 水杨酸处理对苹果轮纹病的抑制效果 $[\mathrm{J}]$. 北方园 艺, 2010, (3) : 147-148.

[13] 汤晓莉 薛红芬, 邓国宾, 等. 水杨酸诱导马铃薯疮痂病抗性 的生理机制研究 [J]. 西南农业学 报, 2010, 23 (6) : 1851-1854.

[14] 李梅, 毕阳, 葛永红, 等. 采前Harpin处理对 “银帝” 甜瓜潜 伏侵染的影响 [J]. 甘肃农业大学学 报, 2005, 40 (2) : 153-156.

[15] Huang Y, Deverall B J, Tang W H. etal. Foliar application of acibenzolar-S-methyl and protection of postharvest rock melons and Hami melons from disease[J]. European Journal of Plant Pathology. 2000, (106) : 651-656.

[16] 曹建康, 姜微波, 赵玉梅. 果蔬采后生理生化实验指导 [M]. 中国轻工业出版社, 2007.

[17] 朱海英, 李人圭, 王隆华, 等. 丝瓜果实发育中木质素代谢及 有关导管分化的生理生化研究 $[J]$. 华东师范大学学 报, 1997, (1):87-94.

[18］曹建康. SA、ASM、INA和柠檬酸对鸭梨果实采后抗病性和品 质的影响 [D]. 北京: 中国农业大学, 2005 .

[19] Yao H J, Tian S P. Effects of pre- and post-harvest application of salicylic acid or methyl jasmonate on inducing disease resistance of sweet cherry fruit in storage. Postharvest Biology and Technology, 2005, 35: 253-262.

[20] Polle A, Tilman T, Seifert F. Apo plastic per-oxidases and lignification in needles of Norway Spruce (Picea abies L) [J]. Plant Physiology, 1994, 106: 53-60.

[21] Davarynejad G H, Zarei M, Nasrabadi M E, et al. Effects of salicylic acid and putrescine on storability, quality attributes and antioxidant activity of plum cv. 'Santa Rosa' [J]. Journal of Food Science and Technology, 2015, 52(4): 2053-2062.

[22] Babalar, M. , Asghari, M., Talaei, A. , \& Khosroshahi, A. Effect ofpre- and postharvest salicylic acid treatment on ethylene production, fungal decay and overall quality of Selva strawberry fruit. Food Chemistry, 2007, 105, 449-453.

[23] J. -P. M é traux. Systemic Acquired Resistance[J]. Brenner' s Encyclopedia of Genetics (Second Edition), 2013, 1(4): 627-629.

[24] Klarzynaski 0, Plesse B, Joubertj M, et al. Linear $\beta$ -1,3-glucanaseare elicitors of defense responses in tobacc1[J]. PlantPhysiol, 2004， 124: 1027- 1037.

[25] Selit rennikoff C P. Antifungal proteins[J]. Apple Environ Microbiol, 2001, 67: 2883-2894. 
[26] 张衍荣, 王小菁, 张晓云, 等. 水杨酸对豇豆枯萎茵的抑制作 用 [J]. 华中农业大学学报, 2006, 25(6) :610-613.

[27] 张正科, 毕阳, 王军节, 等. 采前苯丙噻重氮 (BTH) 处理对厚 皮甜瓜果实防御酶及抗性物质的诱导 $[\mathrm{J}]$. 甘肃农业大学学 报 2006, 41 (6): 122-125.

[28] Felipini R B, Luiz C, Costa M E B, et al. Mode of action of chitosan and ASM for the control of Cercospora leaf spot on table beet [J]. Tropical Plant Pathology, 2015, 40 (3) : 176-183.
[29] 王媛, 杨红玉, 程在全. SA诱导拟南芥对灰霉病的抗性与木 质素含量的关系 [J]. 植物保护, 2007, 4(33) : 50-54.

[30] 张帆, 王友升, 刘晓艳, 等. 采前水杨酸处理对树莓果实咜藏 效果及抗氧化能力的影响 $[\mathrm{J}]$. 食品科 学, 2010, 10 (31) :308-312.

[31] 朱璇. 壳聚糖和BTH对芒果 (Mangifera indica L. ) 果实采后 抗病性和品质的影响 [D]. 中国农业大学, 2007. 